\title{
Factors influencing knowledge and practice of birth preparedness and complication readiness in sub-saharan Africa: a narrative review of cross-sectional studies
}

\author{
Joshua Sumankuuro*, Judith Crockett, Shaoyu Wang
}

School of Community Health, Faculty of Science, Charles Sturt University, Orange, NSW, 2800, Australia

Received: 08October 2016

Accepted: 10 November 2016

*Correspondence:

Joshua Sumankuuro,

E-mail: jsumankuuro@csu.edu.au

Copyright: ( ) the author(s), publisher and licensee Medip Academy. This is an open-access article distributed under the terms of the Creative Commons Attribution Non-Commercial License, which permits unrestricted non-commercial use, distribution, and reproduction in any medium, provided the original work is properly cited.

\begin{abstract}
Birth preparedness and complication readiness $(\mathrm{BP} / \mathrm{CR})$ is a strategy that encourages pregnant women, their families, and communities to effectively plan for births and deal with emergencies, if they occur. The study assessed knowledge on and practice of BP/CR among expectant mothers, partners and community. A systematic search of peer reviewed journals in English language quantitative and qualitative studies published between 2010 and 2015 was undertaken using Primo search, Ovid and Scopus databases. Knowledge of birth preparedness was higher than knowledge of pregnancy danger signs (complication readiness) in many studies, whilst implementation of BP/CR interventions was lower than level of knowledge in all studies. Education, parity, antenatal care visits, age, place of residence and gender were better predictors of knowledge on BP/CR; financial difficulties, employment status, knowledge of danger signs, distance to health facility, the involvement of men and community perceptions were significant determinants of BP/CR practice. Relevant studies are welcomed on the perceptions and challenges of translating BP/CR knowledge into practice in hard-to-reach communities in sub-Saharan Africa.
\end{abstract}

Keywords: Birth preparedness and complications readiness, Factors, Knowledge and practice, Maternal healthcare, Sub-Saharan Africa

\section{INTRODUCTION}

Pregnancy and child birth in many parts of developing countries is a perilous journey, which could result in risk of lifetime disability or death. ${ }^{1}$ Indeed, in the absence of skilled obstetric care, $15 \%$ of all pregnant women will suffer from serious and long term morbidities and disabilities. $^{2}$ Maternal and neonate mortality has decreased worldwide in the last ten years; however, in sub-Saharan Africa, it is still high.,

A study in this region discovered a large number of about $25 \%$ to $33 \%$ of all deaths, among women of reproductive age result from complications of pregnancy or childbirth and an approximate burden of 1,500 maternal deaths every day. ${ }^{4}$ The risk of an African woman dying from complication related to pregnancy or childbirth is 1 in 16 , as compared to 1 in 2,800 in a developed country. ${ }^{5}$ Lifetime risk of mortality among women is 1 in 38 in subSaharan Africa compared to 1 in 3,700 in the developed world. $^{2,6}$

Women and newborns need timely access to skilled care during pregnancy, childbirth, and the postpartum/newborn period. ${ }^{5}$ Too often, access to care is impeded by delays - delays in deciding to seek care, delays in reaching care, and delays in receiving care. ${ }^{3,6-8}$ Identification of these key delays resulted in the development of the birth preparedness and complication readiness approach - the process of planning for a normal 
birth and anticipating danger signs. The Birth Preparedness and Complication Readiness (BP/CR) matrix delineates the roles of mothers, their partners, other family members, healthcare providers and community members involved in pregnancy, childbirth and postpartum period. If the $\mathrm{BP} / \mathrm{CR}$ is successfully implemented, it is anticipated that there will be an improvement to the maternal and neonatal health outcomes. $^{9,10}$

The key components of the birth plan package include: recognition of danger signs, a plan for a birth attendant, identifying place of delivery, and saving money for transport or other costs in case the need arises. ${ }^{5,9}$ In addition, a potential blood donor and a decision-maker (in case of emergencies) need to be identified, considering the unreliable stock of blood banks in most hospitals in sub-Saharan Africa such as Ghana. ${ }^{4,11}$ Complications such as haemorrhage are unpredictable and potentially fatal if timely treatment is not obtained. ${ }^{2,11}$

This makes the BP/CR package a key strategy in subSaharan African countries, where obstetric services are generally poor. ${ }^{3}$ The BP/CR initiative was adopted across sub-Saharan Africa in the early 2000's for the purpose of increasing health facility deliveries soon after the introduction of focused antenatal care (FANC), ${ }^{2,5}$ which replaced the more traditional risk assessment approach (ANC) in many locations. With its emphasis on ensuring the presence of a skilled birth attendant at every child birth and promoting awareness of danger signs, FANC needs to be prioritised and planned for as prime catalyst for achieving $\mathrm{BP} / \mathrm{CR}$. $^{1,10}$ The review focused on the factors influencing the knowledge on and implementation of $\mathrm{BP} / \mathrm{CR}$ in sub-Saharan Africa using findings from original research work.

\section{METHODS}

A narrative approach was adopted for the review. This approach provides a means for developing both interpretive and narrative synthesis from the broader perspective of the experiences of women and men regarding impeding factors affecting their preparedness for birth and complication readiness. ${ }^{12}$ The review method assisted in generating a deeper knowledge of the nature of $\mathrm{BP} / \mathrm{CR}$, rather than just summarising a number of studies. This paper followed the guidelines given in the protocol for conducting a systematic review on BP/CR studies, the PRISMA statement and birth preparedness and complication readiness tools and indicators for maternal and newborn health by JHPIEGO and the WHO. ${ }^{9,13}$

We searched published literature from Primo search, Scopus and Ovid databases. Additionally, it provides insight into the dynamics underlying the findings of the different approaches within the framework of crosssectional design. The method allowed flexibility in the review process, as well as the utilisation of the descriptive phrases and metaphors found within existing studies, to create different perspectives about the world view. The BP/CR Index is a set of six separate indices, one for each level measured - individual, family, community, provider, facility and policy levels. ${ }^{9,13}$ This review considered papers which contained more than two of the following immediate influential factors which determines maternal and neonate health outcomes: (a) knowledge of danger signs; (b) plan for a birth attendant; (c) identifying a blood donor; (d) plan for the place of child birth, and (e) saving money for transport or other costs in case the need arises. ${ }^{9}$ We conducted a search on main databases: Primo search and Scopus, and handsearching of some reference lists obtained within ninemonth period (November, 2015 to July, 2016). On Primo search for instance, the saved search "BPCR" on Charles Sturt University online library ("libaskus" service) produced results for 54 times with same outputs.

\section{Inclusion criteria}

The results from the database search produced a total of 164 papers. However, based on the preconceived focus of the paper, only quantitative and qualitative peer-reviewed original research articles whose setting was in subSaharan Africa were included in this write-up. The BP/CR implementation protocol prepared by Johns Hopkins Program for International Education in Gynaecology and Obstetrics (JHPIEGO) and the WHO, guided the aims of the study. The results of the inclusion and exclusion criteria are summarised in the PRISMA diagram. The study adopted the model by Miltenburg et al as shown below (Table 1). ${ }^{13}$

\section{Exclusion criteria}

Among the 164 articles initially identified, 145 papers were excluded. The excluded papers were either duplicated or found outside sub-Saharan Africa or not a peer-reviewed original article. Full abstract scan of 55 articles were carried out for eligibility from which 10 duplicate studies were excluded. Fifteen papers were excluded after full reading and critically appraising them for eligibility using the critical appraisal format by Young and Solomon. ${ }^{25}$ Repeated studies on the search were excluded. Systematic reviews, gray literature, conference or newspapers were also excluded. Journals that captured neonatal and maternal health care but excluded BP/CR elements were also excluded.

\section{Data review}

The data review was conducted from early November, 2015 to August, 2016 using the following electronic bibliographical databases: Primo Search, Scopus and Ovid and the World Health Organization (WHO) library. A manual search was also carried out using other relevant citations such as in JHPIEGO research on BP/CR. The keywords used for the search were "factors influencing birth preparedness" and "complication readiness" and 
"sub-Saharan Africa". Papers were included if they presented empirical cross-sectional descriptive factors influencing knowledge on and practice of $\mathrm{BP} / \mathrm{CR}$ and were published in English language (Figure 1).

Table 1: Characteristics of included studies.

\begin{tabular}{|c|c|c|c|}
\hline Author & Participants & Methods & BP/CR experiences \\
\hline August $\mathrm{F}$ et $\mathrm{al}^{6}$ & $\begin{array}{l}\mathrm{N}=132 \text { (12 FGDs: } 65 \text { young men } \\
\text { and women, } 67 \text { older men and } \\
\text { women) }\end{array}$ & Qualitative & $\begin{array}{l}\text { Knowledge on the components of } \\
\text { BP/CR }\end{array}$ \\
\hline August $\mathrm{F}$ et $\mathrm{al}^{2}$ & $\mathrm{~N}=756$ recent fathers & Cross-sectional quantitative & $\begin{array}{l}\text { Knowledge on the components of } \\
\text { BP/CR; Male involvement in } \\
\text { BP/CR }\end{array}$ \\
\hline Bayu $\mathrm{H}$ et $\mathrm{al}^{14}$ & $\begin{array}{l}\mathrm{N}=522 \text { second and third trimester } \\
\text { pregnant women who had planned } \\
\text { for institutional delivery }\end{array}$ & Quantitative & $\begin{array}{l}\text { Awareness of danger signs } \\
\text { Predictors of BP/CR }\end{array}$ \\
\hline Bintabara D et al ${ }^{15}$ & $\begin{array}{l}\mathrm{N}=428 \text { women from } 22-33 \text { yrs of } \\
\text { age }\end{array}$ & Quantitative cross-sectional & $\begin{array}{l}\text { Knowledge and determinants of } \\
\text { the practice of BP/CR }\end{array}$ \\
\hline Brazier E et al $^{16}$ & $\begin{array}{l}\mathrm{N}=1,846 \text { heads of households; } \\
2,335 \text { women of reproductive age, } \\
\text { of which } 1,333 \text { had given birth } \\
\text { (within past } 24 \text { months \& } 735 \text { in } \\
\text { past } 5 \text { years) }\end{array}$ & $\begin{array}{l}\text { Quantitative population- } \\
\text { based household survey }\end{array}$ & $\begin{array}{l}\text { Knowledge of danger signs } \\
\text { application of BP/CR protocol }\end{array}$ \\
\hline Debelew GT et $\mathrm{al}^{17}$ & $\mathrm{~N}=3612$ pregnant women & Cross-sectional quantitative & Predictors of BP/CR \\
\hline Dimtsu B et al ${ }^{18}$ & $\mathrm{~N}=220$ women & Cross-sectional descriptive & Knowledge and practice of BP/CR \\
\hline Ekabua JE $^{1}$ & $\mathrm{~N}=800$ women & Quantitative multi-centric & Predictors of BP/ CR \\
\hline Gebre $\mathrm{M}$ et $\mathrm{al}^{8}$ & $\mathrm{~N}=578$ pregnant women & $\begin{array}{l}\text { Quantitative community } \\
\text { based cross-sectional study }\end{array}$ & $\begin{array}{l}\text { Identifying skilled birth } \\
\text { attendants }\end{array}$ \\
\hline Hailu $\mathrm{M}$ et $\mathrm{al}^{7}$ & $\mathrm{~N}=812$ pregnant women & $\begin{array}{l}\text { Quantitative community } \\
\text { based cross -sectional study }\end{array}$ & $\begin{array}{l}\text { Knowledge of BP/CR } \\
\text { components; Low }(17 \%) \\
\text { preparedness for facility birth }\end{array}$ \\
\hline Iliyasu $\mathrm{Z}$ et al $^{19}$ & $\begin{array}{l}\mathrm{N}=400 \text { men whose } \\
\text { wife/wives have ever been pregnant }\end{array}$ & Cross-sectional descriptive & $\begin{array}{l}\text { Awareness and knowledge of } \\
\text { BP/CR components }\end{array}$ \\
\hline Kakaire et $\mathrm{al}^{20}$ & $\begin{array}{l}\mathrm{N}=140 \\
\text { women admitted as emergency } \\
\text { obstetric referrals in ANC, labour } \\
\text { or postpartum }\end{array}$ & Quantitative cross-sectional & Key components of BP/CR \\
\hline Botha $\mathrm{AK}$ et $\mathrm{al}^{21}$ & $\begin{array}{l}\mathrm{N}=15 \text { randomly selected } \\
\text { postpartum mothers during their } \\
\text { most recent labour and delivery }\end{array}$ & Cross-sectional descriptive & $\begin{array}{l}\text { Key components of BP/CR and } \\
\text { postpartum nutrition }\end{array}$ \\
\hline Lawrence $\mathrm{AL}$ et $\mathrm{al}^{5}$ & $\mathrm{~N}=87$ women & $\begin{array}{l}\text { Qualitative } \\
\text { phenomenological study }\end{array}$ & $\begin{array}{l}\text { Awareness and knowledge of } \\
\text { BP/CR }\end{array}$ \\
\hline Lori JR et $\mathrm{al}^{22}$ & $\begin{array}{l}\mathrm{N}=68,6 \text { focus group discussions } \\
\text { with pregnant women attending } \\
\text { ANC at a busy urban hospital in } \\
\text { Ghana }\end{array}$ & $\begin{array}{l}\text { exploratory, qualitative } \\
\text { study design }\end{array}$ & $\begin{array}{l}\text { Knowledge of BP/CR; Impact of } \\
\text { ANC services on skilled care } \\
\text { seeking behaviour among } \\
\text { pregnant women }\end{array}$ \\
\hline Markos D et al ${ }^{4}$ & $\mathrm{~N}=562$ & $\begin{array}{l}\text { Quantitative community } \\
\text { based cross-sectional study }\end{array}$ & $\begin{array}{l}\text { ANC follow-up, knowledge } \\
\text { about key danger signs during } \\
\text { pregnancy/postpartum }\end{array}$ \\
\hline Mbalinda et $\mathrm{al}^{3}$ & $\mathrm{~N}=810$ women & mixed-methods study & Factors influencing BP/CR \\
\hline Pembe $\mathrm{AB}$ et $\mathrm{al}^{23}$ & $\begin{array}{l}\mathrm{N}=1538 \text { women referred from } \\
18 \text { primary levels of care facilities } \\
\text { (in } 13 \text { months' period). }\end{array}$ & Quantitative follow-up & Emergency referrals and BP/CR \\
\hline 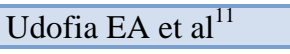 & $\mathrm{N}=483$ childbearing women & Cross-sectional quantitative & Five key areas of BP/CR \\
\hline $\begin{array}{l}\text { Kuganab-Lem RB et } \\
\mathrm{al}^{24}\end{array}$ & $\begin{array}{l}\mathrm{N}=400 \text { postpartum mothers from } \\
40 \text { communities }\end{array}$ & Cross-sectional descriptive & $\begin{array}{l}\text { awareness of birth preparedness } \\
\text { and complication readiness } \\
\text { strategies }\end{array}$ \\
\hline
\end{tabular}




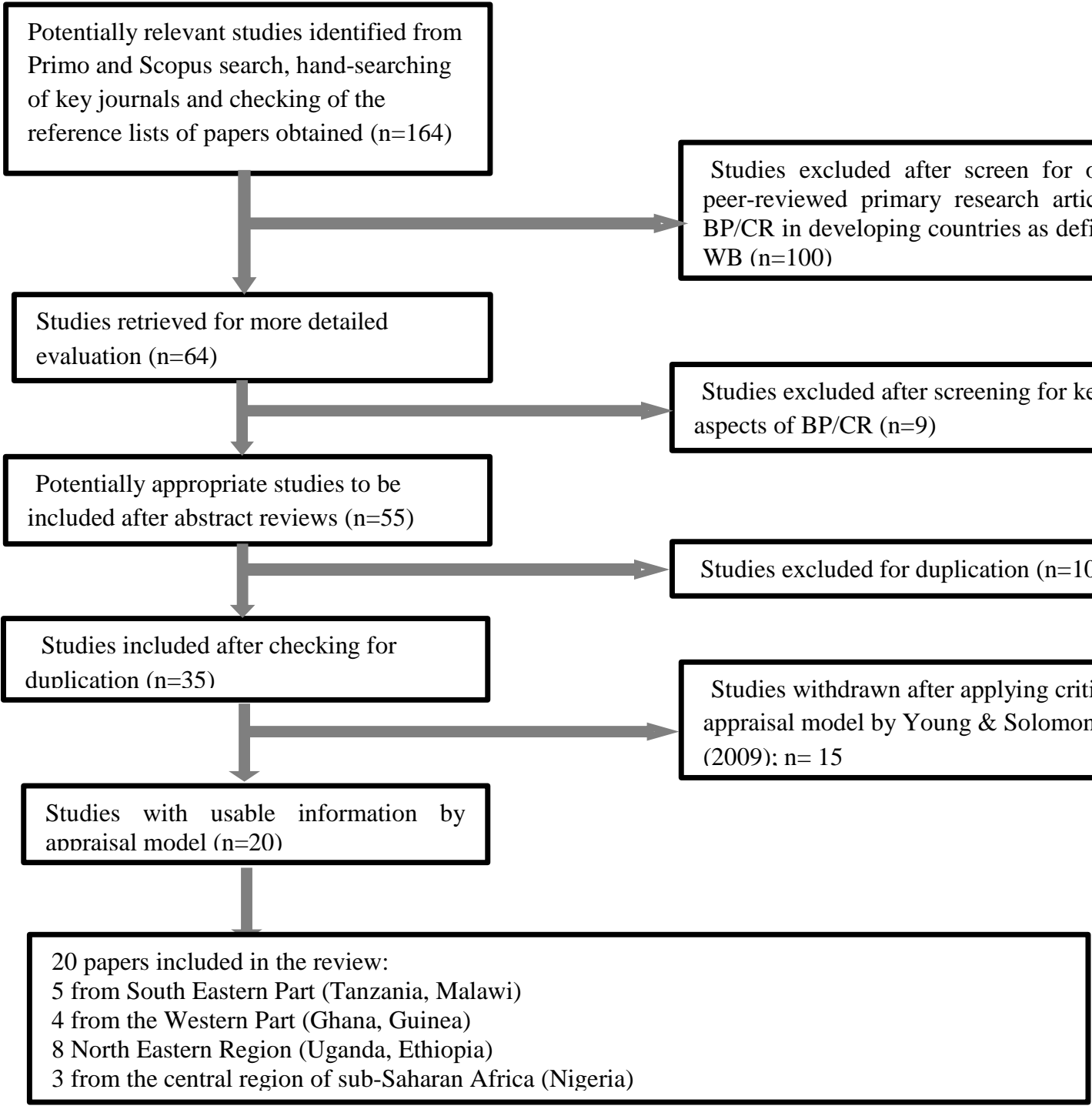

Figure 1: Prisma flow diagram adapted from Miltenburg et al. ${ }^{13}$

\section{RESULTS}

The emerging themes represent the experiences of $\mathrm{BP} / \mathrm{CR}$ in the individual studies reviewed from the 20 articles considered. Synthesis of the studies showed two interconnected themes: a) knowledge of BP/CR key components and b) barriers to implementation of knowledge. Sub-themes were developed through content analysis for easy appreciation.

A summary of the findings grouped together the metaphors and phrases used to describe pregnant women and their families' experiences and practice of BP/CR. Some direct statements and quotations were drawn from the original studies to support the sub-themes. However, the sub-themes were found to run through most of the included studies.

\section{Influences on knowledge of BP/CR}

\section{Educational status of women and their partners}

Research identifies sub-Saharan African countries as having one of the world's lowest adult literacy rates, with less than $50 \%$ of the population of 15 years and above being able to read and write, which is well below the world's average of $84.1 \%{ }^{26}$ Illiteracy among women is highest constituting nearly two-thirds of the illiterate population. ${ }^{6}$ In Guinea, the majority of study participants had never attended school, giving an illiteracy rate of about $79 \%$. In a qualitative study to explore the perception of community members on BP/CR in rural Tanzania found low educational status of citizens, especially women, to have great influence on BP/CR acceptance. ${ }^{6}$ 
Similarly, in Ungogo area of northern Nigeria, men's participation in $\mathrm{BP} / \mathrm{CR}$ and accompaniment/support to antenatal care (ANC) and postnatal care (PNC) were determined by the educational status of the males. Educated males were four times more likely to support in safe motherhood than illiterate counterparts. ${ }^{16}$ Mothers with no formal education but with birth plans were seven times more likely to give birth at home than literate ones in Tigray zone, Ethiopia, and three times more likely to have knowledge on key danger signs in Oromia region. ${ }^{4,14}$ Educational level of mothers was found as significant predictor of knowledge on BP/CR in Mekelle, Ethiopia. ${ }^{4}$ It was better predictor of use of a skilled attendant at birth than uneducated counterparts in Sissala East district, Ghana. ${ }^{24}$ Kaso and Addisse found $69 \%$ of 575 expectant mothers were illiterates in Arsi zone, Ethiopia, and $86.4 \%$ were able to complete at least one antenatal visit. ${ }^{10}$ Among them, $40.2 \%$ and $13 \%$ could mention at least one and two danger signs respectively. About $67 \%$ and $5 \%$ of mothers attained primary and secondary education in Chamwino, Tanzania. Approximately 59\% had knowledge on five key components of BP/CR. ${ }^{15}$ Women who had formal education in Chamwino district, Tanzania were two times more likely to have knowledge on BP/CR than uneducated women. ${ }^{15}$ About $67 \%$ of Malawian mothers who participated in the study had attained primary school education but were unable to identify key danger signs in pregnancy. ${ }^{21}$ A study involving postnatal women in Accra, Ghana, $66 \%$ and $56 \%$ of women and partners respectively had secondary school level education whilst $7 \%$ had no formal education. Among all these mothers, about $69 \%$ had relevant knowledge on BP and $21 \%$ had essential knowledge on danger signs in pregnancy and labour. ${ }^{11}$ In Guinea, 79\% of mothers were illiterate and $31 \%$ had knowledge of BP whilst $14 \%$ had knowledge on CR. ${ }^{16}$

\section{Antenatal visits and knowledge of BP/CR}

In a project in Uganda among mothers who had completed three or more ANC visits; in $5^{\text {th }}$ to $7^{\text {th }}$ months of gestation; and were referred to Mulago hospital, approximately $37 \%$ had essential knowledge on BP/CR. ${ }^{3}$ Nearly 1 in every 3 women were able to mention at least three components of BP/CR. ${ }^{3}$ Another random selection in the same investigation, 1 in every 4 women had knowledge of any of the five key components of BP/CR. ${ }^{3}$ In Oromia region, $15 \%$ of expectant mothers who achieved four or more ANC visits and had knowledge on BP/CR. Hence, women who utilised ANC were eight times more likely to have knowledge on BP and key danger signs in complications in pregnancy and labour. ${ }^{4}$ Evaluating health literacy among ANC attendees in Ghana, women had knowledge on some aspects of danger signs of pregnancy and newborns but were unable to decode ANC lessons and messages communicated. ${ }^{22}$ This contrasted with results from Mikelle area in northern Ethiopia. $^{4}$
Knowledge of BP/CR was higher among women who made three or more ANC visits. ${ }^{4}$ In Salima District of Malawi, mothers who received two or three ANC visits had limited knowledge on birth preparedness only. Birth preparedness was mirrored as purchasing birth kits and $27 \%$ were able to identify three danger signs. ${ }^{21}$ In Malawi, about $54 \%$ of participants had completed eight ANC visits, but could identify only three danger signs. ${ }^{21}$ Most expectant mothers $74 \%$ (out of 575) had completed the recommended number of four or more visits in Chamwino district, although only $17 \%$ commenced care in their first trimester. Fifty-nine percent had knowledge of five components of birth preparedness whilst $69 \%$ could not mention any key danger sign on any of the three stages - pregnancy, labour, postnatal. ${ }^{15}$

\section{Age of mothers and the husband/partner}

The age of expectant mothers has received attention in BP/CR intervention programmes. ${ }^{11}$ Honouring referrals in eventualities of pregnancy are positive steps to receiving skilled care. ${ }^{19,23}$ Assessing effectiveness of maternal referral in Rufiji district, birth preparedness and knowledge on danger signs of complications was low among mothers aged 20 and below. ${ }^{23}$ Thirty-seven percent of them did not also honour referrals. ${ }^{23}$

Similarly, in Chamwino area, the majority of the mothers (53\% of 575) were within the cohort of 21-29 years of age; although more than $70 \%$ of them completed ANC course, $69 \%$ had no knowledge of any danger sign. ${ }^{15}$ In northern Nigeria, higher age $(>30)$ of male partners was a better predictor of participation and support to spouse during maternity care. ${ }^{19}$ They had better awareness on BP although not on CR, than men aged 30 and below. ${ }^{19}$ There were similar findings among 763 multipara mothers with a mean age of 27 at first birth in Guinea. ${ }^{16}$ Only 9\% had knowledge on birth preparedness whilst $10 \%$ could identify few danger signs of complications. ${ }^{16}$ Overall age was a good predictor of knowledge on birth preparedness in many studies but relatively poor predictor on complication readiness. ${ }^{1,7,15}$

\section{Marital status of women}

Marital status was a significant determinant of knowledge on BP/CR among 575 early postnatal mothers in Oromia region, Ethiopia. ${ }^{10}$ About $94 \%$ the of mothers were married among which $40 \%$ had knowledge on at least one danger sign in pregnancy, $42 \%$ on labour and $43 \%$ in the postpartum stage. ${ }^{10}$ Girls in rural Tanzania who conceived before marriage did not attend ANC, had no knowledge on danger signs and were found more likely to give at home due stigmatisation from the community. ${ }^{6}$ A similar study on missed opportunity to give birth at planned health facility in Ethiopia discovered single women were two times more likely to deliver at home than married women. ${ }^{14}$ 


\section{Place of residence}

Assessing awareness of BP/CR strategy in Cross River State of Nigeria, Ekabua, Ekabua, Odusolu, Agan Iklaki and Etokidem observed rural dwellers, had poor knowledge of danger signs and were less likely to use a skilled attendant at birth than mothers who resided in urban areas. ${ }^{1}$ Birth preparedness was also reduced to acquisition of birth kits in rural locations. ${ }^{11}$ There was high awareness of birth preparedness in among postnatal mothers in a hospital study in the capital city of Ghana, but relatively low knowledge on complication readiness among those who travelled over 2 kilometres to access care. $^{11}$ Kugnab-Lem conducted an evaluation of birth preparedness among early postnatal mothers in Sissala East district of northern Ghana and found that 19\% of mothers utilised maternal healthcare for early detection of risks in pregnancy, and $64.2 \%$ had homebirths whilst $35.8 \%$ gave birth at health facility. ${ }^{24}$ Out of 569 ANC attendees in Dunga Fongo- Ethiopia, urban dwellers were found to be seven times more likely to have knowledge of $\mathrm{BP} / \mathrm{CR}$ than the indigenous people. Men who lived in rural areas of Tanzania had low knowledge of birth preparedness and danger signs. ${ }^{2}$ Hence, mothers who lived in rural areas were less likely to have adequate knowledge on birth preparedness and obstetric danger signs than urban dwellers. ${ }^{2}$

\section{Parity}

The number of pregnancies a woman carried to gestation age influenced their knowledge of maternity care particularly with obstetric complications. In south Tigray Zone, Ethiopia, nulliparae mothers were three times more likely to plan for homebirth than multiparous women. ${ }^{14}$ Assessing male involvement in BP/CR in rural Uganda, Kakaire, Kaye and Osinde observed, multiparous mothers were three times more likely to prepare for birth and recognised danger signs than nulliparae. ${ }^{20}$ Botha, Maluwa, Pindani and Bultemeier found similar results in a health facility study with postnatal mothers in Salima district of Malawi. ${ }^{21}$ Multiparous women who utilised ANC throughout the gestation, had low knowledge of danger signs and did not have birth plans except achieving skilled attendant at birth. ${ }^{21}$ Skilled attendant at birth was a main target because of media education on risks associated with homebirths. ${ }^{21}$ In Oromia region, an estimated $99 \%$ of mothers in the study were prepared for birth either at home and health facility. ${ }^{10}$ Ninety percent gave birth at home whilst the remaining $9.2 \%$ prepared for birth and obstetric complications because they had experienced complications, still births or had previous childbirth at health facility. ${ }^{10}$ Women who had four or more childbirths were more likely to prepare for birth and ready for complications. ${ }^{10}$ In south-eastern Ethiopia, Hailu, Gebremariam, Alemseged and Deribe obtained results contrary to other studies. They observed prime mothers had more knowledge on birth preparedness and key danger signs and were more prepared for eventualities than multiparous mothers. ${ }^{7}$

\section{Gender}

In a qualitative study in rural Tanzania involving older men and women and young men and young women in separate group discussions, awareness of birth preparedness and obstetric danger signs was significant among older women than the youth. ${ }^{6}$ Nonetheless, women were more likely to be knowledgeable about BP/CR than men, especially on danger signs. The youth could identify few components of birth preparedness but danger signs of pregnancy and labour. ${ }^{6}$ In Ghana, ANC attendees of up to five visits participated in the study. These mothers contextualised birth preparedness as keeping her body clean and getting a birth kit ready. ${ }^{22}$ Mothers were unable to distinguish discomforts in pregnancy from danger signs. ${ }^{22}$

\section{Poverty, knowledge of danger signs}

Out of 810 mothers surveyed in Mulago hospital in Uganda, 610 were unemployed. ${ }^{3}$ The majority were more than 37 years of age and had had childbirth experience, but only 1 in 4 women had knowledge of key components of BP/CR interventions. ${ }^{3}$ Women who were not in formal employment were less likely to have knowledge of danger signs of pregnancy and labour. ${ }^{3}$ An evaluation among 15 postnatal mothers in a health centre in Malawi found $47 \%$ were basically engaged in subsistence farming. ${ }^{21}$ Cross-examining their knowledge on danger signs, HIV was identified as danger sign in pregnancy, whilst $80 \%$ recounted; backache, lower abdominal pains, oedema of the legs and general body pains for danger signs of labour and delivery. ${ }^{21}$ Despite the knowledge on few danger signs, only 5 out of the 15 women saved money for celebrating their birth but had no plans for complications.

\section{Influences on implementation of birth preparedness and complication readiness plan}

\section{Low levels of ANC}

In south Tigray zone, Ethiopia, 465 mothers planned to have institutional childbirth. ${ }^{14}$ Of this number $29 \%$ instead had homebirths. The results showed homebirthing mothers were among the $72 \%$ who made only one ANC visit in the period of pregnancy. ${ }^{14}$ In Oromia Region, Kaso and Addisse recorded slightly higher figures whereby out of 575 mothers, $86.4 \%$ commenced ANC in $4^{\text {th }}$ to $6^{\text {th }}$ month of gestation, and $91 \%$ of them had homebirths. ${ }^{10}$ In a health facility cross-sectional study involving 483 postnatal mothers in Korle-Bu teaching hospital (Ghana), $90 \%$ of mothers received full course of ANC and $98.9 \%$ of all respondents gave birth supervised by skilled professional. ${ }^{11}$ Overall women who utilise ANC services were more likely to give birth at health facility. 


\section{Husband's involvement}

In a quantitative follow-up in Rufiji district, half of the men $(51 \%)$ did accompanied their wives for ANC; $77 \%$ of the participants' spouses also gave birth in a health facility. ${ }^{2}$ Most men (72\%) had made plans for the baby's naming ceremony, whilst less than a third $(30.8 \%)$, of the 400 men who participated in the study, made plans for the mother's health care, arranged for transportation $(24 \%)$ and child birth care $(23 \%)$ in northern Nigeria. ${ }^{19}$ Kakaire, Kaye and Osinde observed that out of 140 women in rural Uganda, $43 \%$ received spouse support to ANC; $42 \%$ had their husbands looked after children; $25 \%$ received support in doing household chores whilst $68.6 \%$ of the mothers were accompanied by the husbands during labour. ${ }^{20}$ Mothers who received support were more likely to utilise maternity care from health facility. ${ }^{20}$ In communities where knowledge on BP/CR was high, males were more likely to accompany their spouses at ANC and childbirth in Rufiji district of Tanzania., 6,20

\section{Preference for homebirth}

In South Tigray Zone, Ethiopia, about 28.8\% (134 of 465 ) of the participants reported having home delivery because there was no problem during labour at home, whilst fewer mothers asserted it was their family decision. ${ }^{14}$ In Oromia region of Ethiopia, $40 \%$ opted for homebirths because of cost of transport and healthcare bills. ${ }^{10}$ Community stigmatisation was an obvious reason for single mothers' choice of homebirths across many areas. $^{14,18,23}$ Some mothers classified themselves as having precipitate labours and therefore chose homebirth for fear of roadside childbirth in Tanzania. ${ }^{23}$

\section{Family finances}

Evaluating BP/CR among postnatal mothers in Ethiopia, Mbalinda, Nakimuli, Kakaire, Osinde, Kakande and Kaye $^{3}$ found that awareness of danger signs are positive signals to wanting to get ready for complications. About $45 \%$ of mothers had identified place of childbirth, and only $4.9 \%$ had planned to give birth at the nearest health facility. A larger proportion (98\%) planned for homebirths. Inability to afford healthcare bills influenced their decision although $86.4 \%$ had completed three or more ANC visits. ${ }^{3}$ Women who saved for bills and transport were more likely to prepare for birth and its complications and were willing to have childbirth with skilled attendant. ${ }^{3,10}$ However, August, Pembe, Kayombo, Mbekenga, Axemo and Darj obtained contrasting results in a qualitative study in Tanzania. ${ }^{6}$ Participants' knowledge on BP/CR components was as adequate as those of Ethiopia. ${ }^{10}$ The husbands and in-laws translated their knowledge into practice by assuming full responsibility of saving money for complications and ensured pregnant women gave birth supervised by skilled attendant. ${ }^{6}$ Conversely in Okpatu community, Nigeria, high cost of facility birth influenced mothers to choose TBAs' care. ${ }^{5}$ Out of 1,538 mothers who were referred to relevant health facility for expert attention in pregnancy, labour and postpartum stages in Rufiji district (Tanzania), $55 \%$ failed to honour the referral advice, and had homebirths due to cost implications. ${ }^{23}$ Practice of BP/CR were two times greater among women whose spouse were employed in Chamwino district, Tanzania. ${ }^{15}$ Mothers who were also employed were more likely to translate knowledge into skilled care during complications and childbirth. ${ }^{15}$

\section{Community perceptions of $B P / C R$}

In a qualitative study in Tanzania, community members were prepared for birth and had moderate knowledge on some danger signs of complications but could not translate it into practice due to limited autonomy for women decision-making and stigmatisation against unmarried mothers by the community. ${ }^{6}$ In an evaluation on missed opportunities for planned health facility birth in rural Ethiopia; women and families had moderate knowledge on birth preparedness and could mention key danger signs of labour but were unable to act as planned due to limited autonomy from family. Mothers who did not also have full financial autonomy also had homebirths. ${ }^{14}$ Assessing knowledge of men on BP/CR and practice in rural Tanzania, it was observed $53 \%$ could mention some danger signs in pregnancy, $44 \%$ in labour and $35 \%$ in puerperium. ${ }^{2}$ However, $54 \%$ supported their wives to purchase birth kit, $2 \%$ identified facility in case of emergency, and less than one percent identified skilled attendant. ${ }^{2}$ There was low implementation of knowledge on key danger signs in Malawian community because expectant mothers and partners did not receive routine education and counselling from the midwives during ANC. $^{21}$

\section{Community engagement in BP/CR}

Forty percent of mothers in Guinea had a high score on the community exposure index to $\mathrm{BP} / \mathrm{CR}$ during community education sessions on maternal health risks and related obstetric complications issues by the nurses and community health volunteers. ${ }^{16}$ Thirty-four percent of the mothers accomplished three or more preparations in their most recent births. ${ }^{16}$ Despite the awareness and previous preparations, BP knowledge index and practice was $31 \%$, and only $14 \%$ had a high score on CR knowledge index. BP behavioural literacy was also associated with BP knowledge and purchase of birth kits, with less focus on practicing CR. ${ }^{16}$ Out of 400 mothers in Sissala East district, only $4.5 \%$ received community support to effect referrals during obstetric complications. ${ }^{24}$ These findings are similar to those of Tanzania., ${ }^{2,6}$ Community members had high knowledge on BP and low knowledge on few danger signs but had no plans for emergency transport. ${ }^{6}$ Others failed to utilise health facility care because of challengeS in securing the service of transport. ${ }^{6}$ 


\section{Geographical isolation of communities}

The location of communities has been a significant predictor of access to skilled care in many locations. ${ }^{9,14}$ There were health workers available in Tanzania to rescue lives of mothers in obstetric complications, but difficulty reaching the appropriate facility deprived them of the services. ${ }^{6,15}$ In another part of Tanzania, community members had moderate knowledge of BP and key danger signs and planned for health facility childbirth but were unable to fulfil these plans due to long distances and non-availability of transport service, compelling roadside and home childbirths. ${ }^{2,6}$ Others do not utilise antenatal care as a result of distance to health facility. ${ }^{2,6}$ Fifty percent of mothers who participated in a study lived in rural areas with motorcycle as the main mode of travel during maternity care in south-eastern Nigeria. ${ }^{1}$ Although about 23\% mentioned prolonged labour as the commonest complications in the area, some had planned for homebirths due to distance to health facility. ${ }^{1}$

\section{Employment and hidden charges in service delivery}

In Rufiji District, Tanzania, an assessment found financial constraints as a major reason for women and their families not following emergency referrals related to obstetric, demographic and natal/birth, pregnancy complications. ${ }^{23}$ In Ghana it was observed that although the Government's National Health Insurance Scheme (NHIS) covers four antenatal visits and cost of skilled attendant at childbirth, $429(88.8 \%)$ in the given study had made some form of payments during child birth. ${ }^{11}$ Women whose partners were employed and could provide for the pregnancy upkeep were four times more likely to practice utilised skilled childbirth services compared to expectant mothers and husbands were unemployed. ${ }^{11,11,19}$

\section{Knowledge of danger signs}

Awareness of danger signs in pregnancy is an important step to reducing delays in seeking care. ${ }^{9,22}$ A study in rural Tanzania acknowledged the wide knowledge of the participants in matters relating to pregnancy complications. $^{2,6}$ But the project results showed community members did not have essential knowledge of some significant threats during pregnancy including: excessive vaginal bleeding and fits during pregnancy, fever, ruptured membranes, leaking of urine, and prolonged labour. ${ }^{6}$ Conditions such as fits were perceived to be caused by evil spirits, and were therefore treated with traditional medicines. ${ }^{6}$

On men's knowledge of danger signs during pregnancy, only $10 \%$ were able to identify excessive vaginal bleeding as a potential complication. ${ }^{2}$ Overall men in this community have a low level of knowledge of obstetric danger signs. ${ }^{2}$ In Malawi there was adequate knowledge on BP/CR components, based on the responses. ${ }^{21}$ However, most of the danger signs during labour and delivery were not known to the participants, and the majority of women misunderstood the difference in danger signs at every stage of gestation. ${ }^{21}$ These findings are similar to those of Ghana where women who received antenatal care and had adequate knowledge on birth preparedness and few danger signs, yet could not implement the knowledge. It was found challenging to differentiate between common discomforts of pregnancy and emergent signs, when to take prompt action to receive skilled care, and the main reason for $\mathrm{BP} / \mathrm{CR}$ was unclear to them. ${ }^{22}$ Women could not identify danger signs in newborns either. ${ }^{22}$ There was high awareness of BP concept in Cross River State of Nigeria based on the traditional approach to ANC, nonetheless, there was poor knowledge on danger signs, hence, women and families did not make arrangements for any eventualities in pregnancy. ${ }^{1}$ Taking knowledge on danger signs and BP/CR into account, women in Uganda demonstrated there was significant association between danger signs during pregnancy and the postpartum period with birth preparedness. ${ }^{2,20}$ Thus, the greater the knowledge of danger signs, the greater the level of birth preparedness.

\section{Healthcare staff as a challenge}

Midwives and other nurses play a significant role in ensuring all complications and childbirths receive skilled care, particularly in predominantly illiterate communities. ${ }^{1,22}$ In some communities' families and expectant mothers were poorly treated thereby discouraging them from repeated follow-up visits. In Ghana, women lamented being returned home after seeking care during heart palpitation, undue headache and other perceived threats of pregnancy. ${ }^{22}$ Expectant mothers were reported seeking TBAs care and herbalists' consultations before health facility care. ${ }^{22}$ Eight hundred and two expectant mothers were referred from primary health facility to the hospital for childbirth in Rufiji, Tanzania. ${ }^{23}$ Approximately 416 did not comply to the referral advice due to shortage of midwives (3) and doctors (1) in the referral hospital. ${ }^{23}$

These findings were based on the following 20 selected studies by countries across sub-Saharan African region (Figure 2).

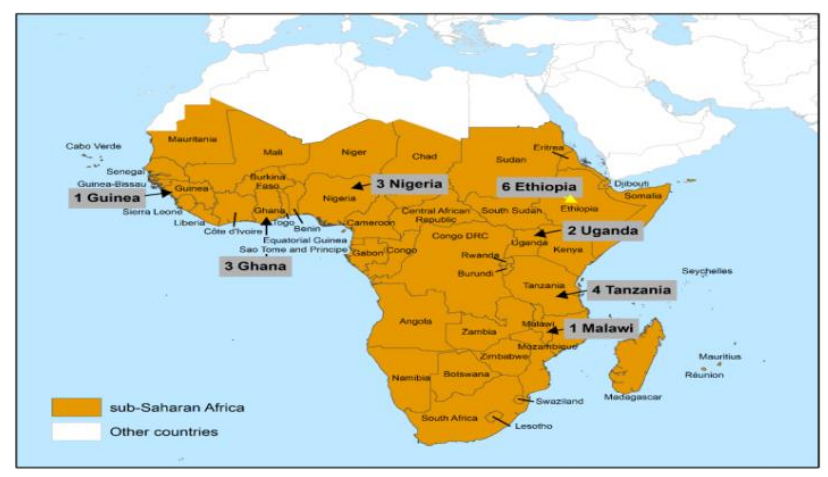

Figure 2: Pictorial view of selected studies in subSaharan African map. 


\section{DISCUSSION}

The paper presents factors influencing knowledge on BP/CR and barriers to knowledge application. The findings indicate that the issues pertaining in getting prepared for birth and complication readiness goes beyond the BP/CR protocol developed by the WHO and JHIPIEGO for monitoring safe motherhood programmes in sub-Saharan Africa. ${ }^{9}$ A combination of educational status, financial status of family, parity, gender, ANC patronage, and marital status influenced mothers' knowledge and practice of BP/CR.

\section{Influences on knowledge of BP/CR}

Knowledge on birth preparedness and key danger signs in pregnancy, delivery and postpartum are found to be a significant influence on utilisation of skilled attendant during in cases of eventualities. ${ }^{20}$ The findings show that education, parity, marital status, age of mother, place of residence (rural and urban) were significant predictors of knowledge on birth preparedness and key danger signs. For example place of residence, educational attainment of mother and parity were better predictors of knowledge on danger signs in pregnancy, labour and postnatal stages in Guinea, Ethiopia, Uganda, Nigeria, Ghana, Malawi and Tanzania. ${ }^{1,2,5,6,20,21,24}$

Thus, multipara women were many times more likely to identify danger signs such as vaginal bleeding than nulliparae. Educated mothers were found to have adequate knowledge on birth preparedness and key danger signs on -pregnancy, labour and postnatal in Ghana, Guinea, Ethiopia, and Tanzania than Malawi, northern Nigeria and Uganda. ${ }^{1,6,8,14,18,19,21,24}$

Uneducated mothers were also more likely to be knowledgeable on key aspects of birth preparedness such as purchase of birth kits and few danger signs in newborns than pregnancy and labour. Multi-parity was also a better predictor of knowledge on birth preparedness and obstetric complications in pregnancy and labour than nulliparous mothers. ${ }^{1-3,10,14,16,19-21,23,24}$ In southern Ethiopia, first time mothers were more prepared for birth but not complications than multiparous women. The limited knowledge on danger signs among first time mothers showed their plans for homebirths. ${ }^{7}$

Gender was key determinant of knowledge on BP/CR. Women were found more likely to have knowledge on $\mathrm{BP} / \mathrm{CR}$ than men. But educated men were more likely to have knowledge on BP than danger signs. ${ }^{20}$ We found that elderly women and men were also more likely to have knowledge on some danger signs than young women and men. But young women had high BP knowledge index than older women. ${ }^{6}$ Expectant mothers who made three or more ANC visits had knowledge on birth preparedness than those who did not make regular ANC visits. ${ }^{2,3,5,6,14,15,24}$
Knowledge on danger signs was limited across all study areas. Women who utilised ANC had knowledge on few key danger signs. ${ }^{10,21}$ Some studies discovered community members and mothers who attended ANC portrayed essential knowledge on some danger signs and birth preparedness but were unable to interpret it. ${ }^{22}$

Men who accompanied their spouses to ANC were found having knowledge on birth preparedness and a few danger signs than their counterparts who did not attend ANC. They were also more likely to support mothers in maternity care. Mothers who were poor were also less likely to have knowledge on danger signs than those who were in formal employment and had financial autonomy and family support. ${ }^{3}$

\section{Barriers to application of knowledge}

Application of knowledge of BP/CR are hindered by multiple factors. Low level of ANC attendance, husband's involvement, family finances, community perception about BP/CR, community support, distance of communities from health facility, knowledge of danger signs, and the job of women and their partners were significant determinants of the implementation of BP/CR strategy. Women who had absolute support from their husbands and had made four or more ANC visits were more likely to utilise skilled care during normal childbirth and complications. ${ }^{1-8,10,11,14-16,18-21,23,24}$

Women who lived close to a health facility or in an urban area utilised skilled care throughout gestation and postnatal than women who live in geographically isolated communities. Men and women who were formally employed were in a better position to afford and utilise skilled care in normal and emergency situations. Across all selected studies, husband's involvement, family finances, knowledge of danger signs, distance to communities and community engagement were better predictors of the implementation of BP/CR knowledge in Ethiopia, Ghana and Tanzania than Malawi, northern Nigeria and Uganda and Guinea. ${ }^{1,2,5-7,10,14-16,18-23}$

Overall, male involvement in birth preparedness and emergency readiness was also low; however, women who received adequate support from partners during ANC and general preparedness for birth and were ready for complication were found translating the knowledge into safe pregnancy and birth outcomes. ${ }^{2}$ Past obstetric history revealed a positive correlation with the knowledge and practice of $\mathrm{BP} / \mathrm{CR} .^{18,27}$ However, increased gravidity/children decreased their preparedness for birth and emergency situations. ${ }^{10}$ Women who had knowledge of at least two key danger signs during previous pregnancy were more likely to be ready for any emergency therein, which is consistent with studies across the sub-region. ${ }^{1,6,27,28}$ In sum, there was no association between knowledge of danger signs during childbirth with birth preparedness. The studies in the sub- 
region clearly show, knowledge on obstetric complication does not translate into practice of BP/CR. ${ }^{2,16,24}$

\section{Intervention}

$\mathrm{BP} / \mathrm{CR}$ programmes have been found as a reliable strategy to reducing avoidable obstetric complications which could lead to loss of lives. Sub-Saharan Africa has been making significant progress although relatively slowly, towards reducing preventable maternal and neonatal deaths through BP/CR. What is lacking among studies of improved interventional designs are systematic reviews of those studies to identify the issues pertaining in the various geographical locations on maternal and neonatal health outcomes. Geographically specific systematic reviews are recommended for improved $\mathrm{BP} / \mathrm{CR}$ interventions in the sub-Saharan region.

\section{Limitations}

As narrative reviews focus on wider coverage of a given perspective, this review has not captured all relevant citations. For instance, the experiences of women during $\mathrm{ANC}$, the issues addressed in ANC education, and the diverse experiences of women in their homes regarding BP/CR were not captured in the study. While the findings cannot be generalised, they provide broad-ranging insight into $\mathrm{BP} / \mathrm{CR}$ in the sub-region, with an emphasis on factors that greatly influence BP/CR.

\section{CONCLUSION}

Interventions promoting BP/CR are intuitively appealing; but the influence of such interventions on women's preparation for birth and their use of institutional childbirth care remains unclear. Research and evaluations related to $\mathrm{BP} / \mathrm{CR}$ interventions have yielded mixed evidence on the efficacy of such interventions. While some studies have indicated that BP/CR interventions are effective at increasing use of skilled delivery care, others, particularly studies of interventions that focused primarily on complications readiness, have shown no positive association with institutional childbirth. Given that knowledge about preparations for birth in some locations is associated with increased preparation, whilst knowledge about complication readiness is not. Further research to explain these different outcomes is critical, particularly if carried out in conjunction with studies that assess the outcome of pregnancies of women who practiced BP and/or CR and those who practise neither.

Funding: No funding sources Conflict of interest: None declared Ethical approval: Not required

\section{REFERENCES}

1. Ekabua JE, Ekabua KJ, Odusolu P, Agan TU, Iklaki CU, Etokidem AJ. Awareness of Birth Preparedness and Complication Readiness in Southeastern
Nigeria. ISRN Obstetrics and Gynecology. 2011;2011:560641.

2. August F, Pembe AB, Mpembeni R, Axemo P, Darj E. Men's Knowledge of Obstetric Danger Signs, Birth Preparedness and Complication Readiness in Rural Tanzania. PLoS One. 2015;10(5).

3. Mbalinda, Nakimuli A, Kakaire O, Osinde MO, Kakande N, Kaye DK. Does knowledge of danger signs of pregnancy predict birth preparedness? A critique of the evidence from women admitted with pregnancy complications. Health Research Policy and Systems. 2014;12(1):60.

4. Markos D, Bogale D. Birth preparedness and complication readiness among women of child bearing age group in Goba Woreda, Oromia Region, Ethiopia. BMC Prengnancy and Childbirth. 2014; 14:282.

5. Lawrence AL, Jimmy JA, Okoye V, Abdulraheem A, Igbans RO, Uzere M: Birth Preparedness and Complication Readiness among Pregnant Women in Okpatu Community, Enugu State, Nigeria. Int J of Innovation and Applied Studies. 2015;11(3):644-9.

6. August F, Pembe AB, Kayombo E, Mbekenga C, Axemo P, Darj E: Birth preparedness and complication readiness - a qualitative study among community members in rural Tanzania. Global Health Action. 2015;8:10.3402/gha.v3408.26922.

7. Hailu M, Gebremariam A, Alemseged F, Deribe K. Birth Preparedness and Complication Readiness among Pregnant Women in Southern Ethiopia. PLoS One. 2011;6(6).

8. Gebre M, Gebremariam A, Abebe TA. Birth Preparedness and Complication Readiness among Pregnant Women in Duguna Fango District, Wolayta Zone, Ethiopia. PloS one. 2015;10(9):e0137570.

9. Del Barco R: Monitoring birth preparedness and complication readiness. Tools and indicators for maternal and newborn health. 2004.

10. Kaso M, Addisse M. Birth preparedness and complication readiness in Robe Woreda, Arsi Zone, Oromia Region, Central Ethiopia: a cross-sectional study. Reproductive Health. 2014;11(1):55.

11. Udofia EA, Obed SA, Calys-Tagoe BNL, Nimo KP: Birth and Emergency Planning: A Cross Sectional Survey of Postnatal Women at Korle Bu Teaching Hospital, Accra, Ghana. Afr J Reproductive Health. 2013;17(1):27-40.

12. Liberati A, Altman DG, Tetzlaff J, Mulrow C, Gøtzsche PC, Ioannidis JP, Clarke M, Devereaux P, Kleijnen J, Moher D. The PRISMA statement for reporting systematic reviews and meta-analyses of studies that evaluate health care interventions: explanation and elaboration. Ann internal medicine. 2009;151(4):W65-94.

13. Miltenburg AS, Roggeveen Y, van Elteren M, Shields L, Bunders J, van Roosmalen J, Stekelenburg J. A protocol for a systematic review of birth preparedness and complication readiness programs. Systematic reviews. 2013;2(1):11. 
14. Bayu H, Fisseha G, Mulat A, Yitayih G, Wolday M: Missed opportunities for institutional delivery and associated factors among urban resident pregnant women in South Tigray Zone, Ethiopia: a community-based follow-up study. Global Health Action. 2015, 8:10.3402/gha.v3408.28082.

15. Bintabara D, Mohamed MA, Mghamba J, Wasswa $\mathrm{P}$, Mpembeni RN. Birth preparedness and complication readiness among recently delivered women in chamwino district, central Tanzania: a cross sectional study. Reproductive health. 2015;12(1):44.

16. Brazier E, Fiorentino R, Barry S, Kasse Y, Millimono S. Rethinking How to Promote Maternity Care-Seeking: Factors Associated With Institutional Delivery in Guinea. Health care for women international. 2014;35(7-9):878-95.

17. Debelew GT, Afework MF, Yalew AW. Factors affecting birth preparedness and complication readiness in Jimma Zone, Southwest Ethiopia: a multilevel analysis. The Pan African Medical J. 2014;19:272.

18. Dimtsu B, Bugssa G. Assessment of knowledge and practice towards birth preparedness and complication readiness among women in Mekelle, Northern Ethiopia: descrptive crossectional. Int $\mathbf{J}$ of Pharmaceutical Sciences and Research. 2014;5(10):4293-301.

19. Iliyasu Z, Abubakar IS, Galadanci HS, Aliyu MH. Birth Preparedness, Complication Readiness and Fathers' Participation in Maternity Care in a Northern Nigerian Community. Afr J Reproductive Health. 2010;14(1):21-32.

20. Kakaire, Kaye DK, Osinde MO. Male involvement in birth preparedness and complication readiness for emergency obstetric referrals in rural Uganda. Reproductive Health. 2011;8(1).
21. Botha AK, Maluwa A, Pindani M, Bultemeier K. Birth preparedness and complication readiness among postnatal mothers in Malawi. Health. 2013:2013.

22. Lori JR, Dahlem CHY, Ackah JV, Adanu RMK: Examining Antenatal Health Literacy in Ghana. J Nursing Scholarship. 2014;46(6):432-40.

23. Pembe AB, Carlstedt A, Urassa DP, Lindmark G, Nyström L, Darj E. Effectiveness of maternal referral system in a rural setting: a case study from Rufiji district, Tanzania. BMC Health Services Research. 2010;10:326.

24. Kuganab-Lem RB, Dogudugu R, Kanton L. Birth Preparedness and Complication Readiness: A Study of Postpartum Women in a Rural District of Ghana. Public Health Research. 2014;4(6):225-33.

25. Young JM, Solomon MJ. How to critically appraise an article. Nature Clinical Practice Gastroenterology \& Hepatology. 2009;6(2):82-91.

26. UNESCO: Adult and youth literacy. Institute for Statistics 2013.

27. Gebre M, Gebremariam A, Abebe TA. Birth Preparedness and Complication Readiness among Pregnant Women in Duguna Fango District, Wolayta Zone, Ethiopia. PLoS One. 2015;10(9).

28. Mbalinda SN, Nakimuli A, Kakaire O, Osinde MO, Kakande N, Kaye DK. Does knowledge of danger signs of pregnancy predict birth preparedness? A critique of the evidence from women admitted with pregnancy complications. Health Research Policy and Systems. 2014;12:60.

Cite this article as: Sumankuuro J, Crockett J, Wang $\mathrm{S}$. Factors influencing knowledge and practice of birth preparedness and complication readiness in subsaharan Africa: a narrative review of cross-sectional studies. Int J Community Med Public Health 2016;3:3297-307. 